\title{
DECAY OF SOLUTIONS OF A SYSTEM OF \\ NONLINEAR KLEIN-GORDON EQUATIONS
}

\author{
JOSÉ FERREIRA \\ Institute of Mathematics, UFRJ \\ P.0.Box 68530, Rio de Janeiro, RJ, Brasil \\ and

\section{GUSTAVO PERLA MENZALA} \\ National Laboratory for Scientific Computation \\ LNCC/CNPq, Lauro Muller 455 \\ 22290, Botafogo, Rio de Janeiro, RJ, Brasil and \\ Institute of Mathematics, UFRJ \\ P.0.Box 68530, Rio de Janeiro, RJ, Brasil \\ (Received November 20, 1985)
}

ABSTRACT. We study the asymptotic behavior in time of the solutions of a system of nonlinear Klein-Gordon equations. We have two basic results: First, in the $L^{\infty}\left(\mathbb{R}^{3}\right)$ norm, solutions decay like $0\left(t^{-3 / 2}\right)$ as $t \rightarrow+\infty$ provided the initial data are sufficiently small. Finally we prove that finite energy solutions of such a system decay in local energy norm as $t \rightarrow+\infty$.

KEY WORDS AND PHRASES. Nonlinear Klein-Gordon equations, decay, local energy, uniform decay.

1980 AMS SUBJECT CLASSIFICATION CODE. 35L70.

\section{INTRODUCTION}

Our main purpose in this work will be to study time decay properties of solutions of the nonlinear system of Klein-Gordon equations

$$
\begin{aligned}
& \square u+m^{2} u+g^{2} u v^{2}=0 \\
& \square v+\sigma^{2} v+g^{2} v u^{2}=0
\end{aligned}
$$

where $x$ runs in $\mathbb{R}^{3}$ and $t \geqq 0$. Here $\square$ denotes the d'Alembertian operator i.e. $\square=\frac{\partial^{2}}{\partial t^{2}}-\Delta$ and $\Delta$ is the usual Laplacian operator. In (1.1)-(1.2), $\mathrm{m}, \sigma$ and $\mathrm{g}$ are positive constants. Such systems of interacting relativistic (scalar) fields were suggested by a number of authors in the last two decades, among them we can mention $I$. Segal [1], K. Jbrgens [2] and more recently, V.G. Makhankov [3]. 
In section 3 we consider solutions of (1.1)-(1.2) in a suitable Banach space $X$ and

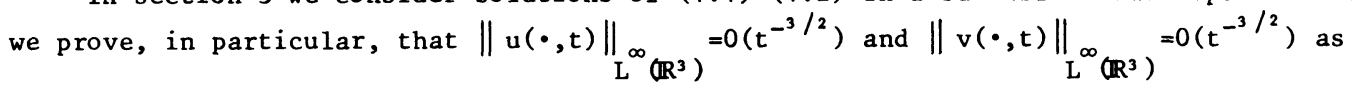
$t \rightarrow+\infty$, provided the initial data is small enough in an appropriate sense. This, seems to be the best possible rate of decay (in the norm \|\|$_{L}$ ) for finite energy solutions of system (1.1)-(1.2). In order to obtain our result we use techniques which are essentially in the framework of contraction type notions together with known facts of the linear Klein-Gordon equation in three dimensional space.

In section 4 we study the local energy behavior as $t \rightarrow+\infty$ for finite energy solutions of (1.1)-(1.2). The important work of C. Morawetz [4] was the starting point for our analysis in this section. Appropriate adaptations of [4] as well as the work of W.A. Strauss [5] to our case were needed. Unfortunately, we could not find the precise rate of decay in this case, which we suspect should be $0\left(t^{-1}\right)$.

\section{NOTATION AND PRELIMINARIES}

In what follows we shall use standard notation: By $\mathrm{L}^{\mathrm{p}}\left(\mathbb{R}^{3}\right), 1 \leqq \mathrm{p}<\infty$ we denote the space of functions in $\mathbb{R}^{3}$ whose pth powers are integrable, with the norm $\|\left. f\right|_{L} ^{p}=\int|f(x)|^{p} d x$ and by $L^{\infty}\left(\mathbb{R}^{3}\right)$ we denote the space of measurable essentially bounded functions in $\mathbb{R}^{3}$, with the norm $\|\mathrm{f}\|_{\mathrm{L}}=e s s \sup |\mathrm{f}(\mathrm{x})|$. From now on, an integral sign to which no domain is attached will be understood to be taken over all space $\mathbb{R}^{3}$. We denote by grad u the gradient of $u$ (in space variables) and $\mid$ grad $\left.u\right|^{2}=\sum_{j=1}^{3}\left(\frac{\partial u}{\partial x_{j}}\right)^{2}$. The radial derivative (with respect to the origem) wil $\}_{3}$ be denoted by $u_{r}=\frac{x}{r} \cdot \operatorname{grad} u$ where $r=|x|$. The Laplacian operator is denoted by $\Delta=\sum_{j=1} \frac{\partial^{2}}{\partial x_{j}^{2}}$. For any positive integer $k$ and $1 \leqq s \leqq \infty$ we consider the Sobolev space $W^{k, s}\left(\mathbb{R}^{3}\right)$ of (classes of) functions in $L^{S}\left(\mathbb{R}^{3}\right)$ which together with their partial derivatives up to order $k$ belong to $L^{S}\left(\mathbb{R}^{3}\right)$. The norm in $W^{k, s}\left(\mathbb{R}^{3}\right)$ will be denoted by \|\|$_{W} k, s$.

In case $s=2$ we shall write $H^{k}\left(\mathbb{R}^{3}\right)$ instead of $W^{k, 2}\left(\mathbb{R}^{3}\right)$. From now on, in order to simplify the notation we will denote by $C$ various constants (which may vary line to line). All functions consider in this paper are real-valued.

Since the system (2.2)-(1.2) is reversible in time, we shall perform our estimates only for $t>0$ and the same conclusion will be true for $t<0$. Most of the lemmas,especially in section 4, are proved only for the case in which the initial data at $t=0$ belongs to $C_{0}^{\infty}\left(\mathbb{R}^{3}\right)$ (that is, the space of $C^{\infty}$ functions defined in $\mathbb{R}^{3}$ with compact support). By a standard approximation procedure the same conclusion will be true for finite energy solutions.

Let us recall briefly some known facts concerning the linear problem: Consider the Klein-Gordon equation

$$
\begin{aligned}
& \square \omega+m^{2} \omega=0, \quad x \text { e } \mathbb{R}^{3}, t e(0, \infty) \\
& \omega(x, 0)=\phi_{1}(x), \omega_{t}(x, 0)=\phi_{2}(x)
\end{aligned}
$$

where $\square=\frac{\partial^{2}}{\partial t^{2}}-\Delta$ and $m>0$. Then, we have the following estimate (see [6]): 


$$
\|\omega(\cdot, t)\|_{L} \leqq C(1+t)^{-3 / 2}\left[\left\|\phi_{1}\right\|_{W} 3,1+\left\|\phi_{2}\right\|_{W} 2,1\right]
$$

provided the initial data $\left(\phi_{1}\right.$ and $\left.\phi_{2}\right)$ belong, say to $C_{0}^{\infty}\left(\mathbb{R}^{3}\right)$.

Now, consider the inhomogeneous Klein-Gordon equation

$\square u+m^{2} u=F(x, t), x \in \mathbb{R}^{3}, t$ e $[0, \infty)$

$$
u(x, 0)=0=u_{t}(x, 0)
$$

where $F$ e $L^{1}\left(0, T ; H^{3}\left(\mathbb{R}^{3}\right) \cap W^{2}, 1\left(\mathbb{R}^{3}\right)\right)$, then using Duhamel's principle and (2.2) we obtain that

$$
\|u(\cdot, t)\|_{L} \leqq C \int_{0}^{t}(1+|t-s|)^{-3 / 2}\|F(\cdot, s)\|_{W^{2,1}} d s
$$

and

$$
\|u(\cdot, t)\|_{H^{3}} \leqq c \int_{0}^{t}\|F(\cdot, s)\|_{H^{3}} d s
$$

Let us define the space of functions which we will be using in the next section: Let $\omega(x, t)$ be such that, for each $t$ we have that $\omega(\cdot, t)$ e $H^{3}\left(\mathbb{R}^{3}\right)$. We consider the norm \|\|$_{D}$ defined by

$$
\|\omega\|_{D}^{2}=\sup _{t \geqq 0}\left[\|\omega(\cdot, t)\|_{H^{3}}^{2}+(1+t)^{3}\|\omega(\cdot, t)\|_{W^{1, \infty}}^{2}\right]
$$

Let

$$
X=\left\{(u, v) \text { such that } u(\cdot, t), v(\cdot, t) \text { e } H^{3}\left(\mathbb{R}^{3}\right) \text { and }\|u\|_{D}<+\infty,\|v\|_{D}<+\infty\right\}
$$

In $X$ we consider the norm $\|(u, v)\|^{2}=\|u\|_{D}^{2}+\|v\|_{D}^{2}$. Clearly, $x$ is a Banach space with the norm $\|(\cdot, \cdot)\|$. Now, we indicate some simple lemmas which will be use in the next section

LEMMA 1. Let $\left(u_{1}, v_{1}\right),\left(u_{2}, v_{2}\right)$ e $X$, then

$$
\begin{aligned}
\left\|u_{1} v_{1}^{2}-u_{2} v_{2}^{2}\right\|_{H^{3}} & +\left\|u_{1} v_{1}^{2}-u_{2} v_{2}^{2}\right\|_{W^{3}, 1} \leqq c\left\|u_{1}-u_{2}\right\|_{D}\left[\left\|v_{1}\right\|_{W}^{2}{ }^{1, \infty}+\left\|v_{1}\right\|_{W^{1, \infty}}\left\|v_{1}\right\|_{H^{3}}\right]+ \\
& +c\left\|v_{1}-v_{2}\right\|_{D}\left[\| u _ { 2 } \| _ { W } { } ^ { 1 , \infty } \left\{\left\|v_{1}\right\|_{D}+\left\|v_{2}\right\|_{D}+\right.\right. \\
& \left.+\left\|u_{2}\right\|_{H^{3}}\left\{\left\|v_{1}\right\|_{W}{ }_{W}+\infty v_{2} \|_{W}{ }_{W}\right\}\right]
\end{aligned}
$$

PROOF. Since $H^{3}\left(\mathbb{R}^{3}\right)$ is an algebra, then, for each $t, u_{1} v_{1}^{2}-u_{2} v_{2}^{2}$ e $H^{3}\left(\mathbb{R}^{3}\right)$. The triangle inequality implies

$$
\left\|u_{1} v_{1}^{2}-u_{2} v_{2}^{2}\right\|_{H^{3}} \leqq\left\|\left(u_{1}-u_{2}\right) v^{2}\right\|_{H^{3}}+\left\|u_{2}\left(v_{1}^{2}-v_{2}^{2}\right)\right\|_{H^{3}}
$$

Using the Leibnitz's rule and the imbedding $H^{3}\left(\mathbb{R}^{3}\right) \subseteq L^{\infty}\left(\mathbb{R}^{3}\right)$ we obtain 
$\left\|\left(u_{1}-u_{2}\right) v^{2}\right\|_{H^{3}} \leqq C\left\|u_{1}-u_{2}\right\|_{D}\left[\left\|v_{1}\right\|_{W}^{2}{ }_{W}^{1, \infty}+\left\|v_{1}\right\|_{W}{ }_{W}, \infty v_{1} \|_{H^{3}}\right]$

and

$$
\begin{aligned}
\left\|u_{2}\left(v_{1}^{2}-v_{2}^{2}\right)\right\|_{H^{3}} & \leqq C\left\|v_{1}-v_{2}\right\|_{D}\left[\left\|u_{2}\right\|_{W^{1, \infty}}\left\{\left\|v_{1}\right\|_{D}+\left\|v_{2}\right\|_{D}\right\}+\left\|u_{2}\right\|_{H^{3}}\left\{\left\|v_{1}\right\|_{W^{1, \infty}}+\right.\right. \\
& \left.\left.+\left\|v_{2}\right\|_{W^{1}, \infty}\right\}\right]
\end{aligned}
$$

which together with (2.6) implies that we got the desired bound for the first term on the left hand side of the lemma. The estimate for the term $\left\|u_{1} v_{1}^{2}-u_{2} v_{2}^{2}\right\|_{W}, 1$ can be done
similarly.

LEMMA 2. Let $q \geqq 1, r>0$ such that $r q>1$, then, for any $t>0$ we have

$$
\int_{0}^{\infty}(1+|t-s|)^{-r}(1+s)^{-r q} d s \leqq C(1+t)^{-r}
$$

PROOF. See [7].

\section{DECAY AS $t \rightarrow+\infty$ FOR SMALL DATA}

In this section we present a result concerning the asymptotic behavior for solutions of (1.1)-(1.2) in the space $X$ and small initial data.

LEMMA 3. Let $\left(u_{1}, v_{1}\right),\left(u_{2}, v_{2}\right)$ e $X$ and $\rho>0$. Suppose that $\left\|\left(u_{1}, v_{1}\right)\right\| \mid \leqq \rho,\|\|\left(u_{2}, v_{2}\right) \| \leqq \rho$. Define the nonlinear maps $\mathrm{N}_{\mathrm{m}}$ and $\mathrm{N}_{\sigma}$ by

$$
\begin{aligned}
& N_{m}\left[u_{j}, v_{j}\right](x, t)=-g^{2} \int_{0}^{t} \int_{m}(x-y, t-s) u_{j} v_{j}^{2} d y d s \\
& N_{\sigma}\left[v_{j}, u_{j}\right](x, t)=-g^{2} \int_{0}^{t} R_{\sigma}(x-y, t-s) v_{j} u_{j}^{2} d y d s
\end{aligned}
$$

$j=1,2$, where $R_{m}$ and $R_{\sigma}$ denote the Riemann functions associated with the linear KleinGordon operator $\square+\mathrm{m}^{2} I$ and $\square+\sigma^{2} I$ respectively. Then

a) $\left\|N_{m}\left[u_{1}, v_{1}\right]-N_{m}\left[u_{2}, v_{2}\right]\right\|_{D} \leqq C \rho^{2}\left\|\left(u_{1}-u_{2}, v_{1}-v_{2}\right)\right\|$

and

b) $\left\|N_{\sigma}\left[v_{1}, u_{1}\right]-N_{\sigma}\left[v_{2}, u_{2}\right]\right\|_{D} \leqq C \rho^{2}\left\|\left(u_{1}-u_{2}, v_{1}-v_{2}\right)\right\|$

PROOF. Since $H^{3}\left(\mathbb{R}^{3}\right)$ is an algebra then it follows that for each $t, u_{1} v_{1}^{2}-u_{2} v_{2}^{2} e H^{3}\left(\mathbb{R}^{3}\right)$. Using the definition and (2.4) we obtain, for each $t$ :

$$
\left\|N_{m}\left[u_{1} v_{1}\right]-N_{m}\left[u_{2}, v_{2}\right]\right\|_{H^{3}} \leqq C \int_{0}^{t}\left\|u_{1} v^{2}-u_{2} v_{2}^{2}\right\|_{H^{3}} d s
$$

By lemma 1 it follows that

$$
\begin{aligned}
& \int_{0}^{t}\left\|u_{1} v_{1}^{2}-u_{2} v_{2}^{2}\right\|_{H^{3}} d s \leqq C\left\|u_{1}-u_{2}\right\|_{D} \int_{0}^{t}\left[\left\|v_{1}\right\|_{W^{1, \infty}}^{2}+\left\|v_{1}\right\|_{W^{1, \infty}}\left\|v_{1}\right\|_{H^{3}}\right] d s+ \\
& +C\left\|v_{1}-v_{2}\right\|_{D}\left[\left\|v_{1}\right\|_{D}+\left\|v_{2}\right\|_{D}\right] \int_{0}^{t}\left\|u_{2}\right\|_{W} 1, \infty d s+C\left\|v_{1}-v_{2}\right\|_{D}\left\|u_{2}\right\|_{D} \int_{0}^{t}\left[\left\|v_{1}\right\|_{W}{ }_{W}+\infty\right. \\
& \left.+\left\|v_{2}\right\|_{W}{ }_{1, \infty}\right] d s \leqq C\left\|u_{1}-u_{2}\right\|_{D}\left\|v_{1}\right\|_{D}^{2}+C\left\|v_{1}-v_{2}\right\|_{D}\left[\left\|v_{1}\right\|_{D}+\left\|v_{2}\right\|_{D}\right]\left\|u_{2}\right\|_{D}
\end{aligned}
$$


Thus

$$
\left\|\mathrm{N}_{\mathrm{m}}\left[\mathrm{u}_{1}, \mathrm{v}_{1}\right]-\mathrm{N}_{\mathrm{m}}\left[\mathrm{u}_{2}, \mathrm{v}_{2}\right]\right\|_{\mathrm{H}^{3}} \leqq C \rho^{2}\left[\left\|\mathrm{u}_{1}-\mathrm{u}_{2}\right\|_{\mathrm{D}}+\left\|\mathrm{v}_{1}-\mathrm{v}_{2}\right\|_{\mathrm{D}}\right]
$$

Using the definition and (essentially) (2.3) it follows that

$$
\left\|N_{m}\left[u_{1}, v_{1}\right]-N_{m}\left[u_{2}, v_{2}\right]\right\|_{W} 1, \infty \leq C \int_{0}^{t}(1+|t-s|)^{-3 / 2}\left\|u_{1} v^{2}-u_{2} v_{2}^{2}\right\|_{W} 3,1 d s
$$

By lemmas 1 and 2 we deduce that

$$
\begin{aligned}
& \int_{0}^{t}(1+|t-s|)^{-3 / 2}\left\|u_{1} v_{1}^{2}-u_{2} v_{2}^{2}\right\|_{W}, 1 d s \leqq\left\{C\left\|u_{1}-u_{2}\right\|_{D}\left\|v_{1}\right\|_{D}^{2}+\right. \\
& \left.\quad+C\left\|v_{1}-v_{2}\right\|_{D}\left[\left(\left\|v_{1}\right\|_{D}+\left\|v_{2}\right\|_{D}\right)\left\|u_{2}\right\|_{D}\right]\right\} \int_{0}^{t}(1+t-s)^{-3 / 2}(1+s)^{-3 / 2} d s \\
& \quad \leqq C(1+t)^{-3 / 2}\left[\left\|v_{1}\right\|_{D}^{2}\left\|u_{1}-u_{2}\right\|_{D}+\left\|v_{1}-v_{2}\right\|_{D}\left\|u_{2}\right\|_{D}\left\{\left\|v_{1}\right\|_{D}+\left\|v_{2}\right\|_{D}\right\}\right]
\end{aligned}
$$

Consequent $1 \mathrm{y}$

$$
(1+t)^{3 / 2}\left\|N_{m}\left[u_{1}, v_{1}\right]-N_{m}\left[u_{2}, v_{2}\right]\right\|_{W} 1, \infty \leqq C \rho^{2}\left[\left\|u_{1}-u_{2}\right\|_{D}+\left\|v_{1}-v_{2}\right\|_{D}\right]
$$

Combining (3.3) with (3.4) we conclude item a). The proof of item b) is done exactly in the same fashion.

LEMMA 4. Let $u_{0}(x, t), v_{0}(x, t)$ be solutions of the free Klein-Gordon equations $\square u_{0}+m^{2} u_{0}=0$ and $\square v_{0}+\sigma^{2} v_{0}=0$ respectively with initial data at time $t=0$ so that $\left(u_{0}, v_{0}\right) e X$. Let us consider the sequence $\left\{\left(u_{(n)}, v_{(n)}\right)\right\}_{n=0}^{\infty}$ defined by $\left(u_{(0)}, v_{(0)}\right)=\left(u_{0}, v_{0}\right)$

$$
\begin{aligned}
& u_{(n+1)}=u_{0}+N_{m}\left[u_{(n)}, v_{(n)}\right] \\
& v_{(n+1)}=v_{0}+N_{\sigma}\left[v_{(n)}, u_{(n)}\right]
\end{aligned}
$$

for $n=1,2, \ldots$ where $N_{m}$ and $N_{\sigma}$ were defined in (3.1). Then $\left(u_{(n+1)}, v_{(n+1)}\right)$ e $X$ for all $\mathrm{n}=0,1,2, \ldots$

PROOF. The proof is done by induction. It is enough to prove that $\left(\mathrm{N}_{\mathrm{m}}\left[\mathrm{u}_{(\mathrm{n})}, \mathrm{v}_{(\mathrm{n})}\right], \mathrm{N}_{\sigma}\left[\mathrm{v}_{(\mathrm{n})}, \mathrm{u}_{(\mathrm{n})}\right]\right)$ e X provided that $\left(\mathrm{u}_{(\mathrm{n})}, \mathrm{v}_{(\mathrm{n})}\right)$ e X. But this was already done during the proof of lemma 3. Consequently the conclusion of the lemma holds.

Now let $u_{0}$ and $v_{o}$ as in lemma 4 with initial data

$$
u_{0}(x, 0)=\phi_{1}(x), \quad \frac{\partial u_{0}}{\partial t}(x, 0)=\phi_{2}(x),
$$

$v_{0}(x, 0)=\psi_{1}(x)$ and $\frac{\partial v_{o}}{\partial t}(x, 0)=\psi_{2}(x)$ such that $\phi_{j}, \psi_{j}$ e $C_{0}^{\infty}\left(\mathbb{R}^{3}\right), j=1,2$. Using (2.2) we can estimate the norm $\left\|\left(u_{0}, v_{0}\right)\right\|$, say $\left\|\left(u_{0}, v_{0}\right)\right\| \leq_{0}$ where 


$$
\begin{aligned}
0<\rho_{\mathrm{O}} & =\mathrm{C}\left[\left\|\phi_{1}\right\|_{\mathrm{H}^{3}}+\left\|\phi_{1}\right\|_{\mathrm{W}^{4}, 1}+\left\|\phi_{2}\right\|_{\mathrm{H}^{2}}+\left\|\phi_{2}\right\|_{\mathrm{W}^{3,1}}+\left\|\psi_{1}\right\|_{\mathrm{H}^{3}}+\left\|\psi_{2}\right\|_{\mathrm{W}^{4,1}}+\right. \\
& \left.+\left\|\psi_{2}\right\|_{\mathrm{H}^{2}}+\left\|\psi_{2}\right\|_{\mathrm{W}^{3,1}}\right]
\end{aligned}
$$

On the other hand, let us choose $\tilde{\rho}>0$ small enough so that $\tilde{\rho}^{2} \leqq \frac{1}{2 \sqrt{2}}$ c where $C>0$ is the constant which appears in the right hand side of inequality (3.2).

THEOREM 1 (Decay for small data). Let $\phi_{j}, \psi_{j}$ e $C_{0}^{\infty}\left(\mathbb{R}^{3}\right), j=1,2$ be chosen so that $0<\rho_{0} \leqq \frac{\tilde{\rho}}{2}$. Then, the sequence of sucessive approximations $\left\{\left(u_{(n)}, v(n)\right\}_{n=0}^{\infty}\right.$ defined in Lemma 3 converges to a pair $(u, v)$ e $X$, which is a solution of $(1.1)-(1.2)$ such that

$$
u(x, 0)=\phi_{1}(x), u_{t}(x, 0)=\phi_{2}(x), v(x, 0)=\psi_{1}(x), \quad v_{t}(x, 0)=\psi_{2}(x) .
$$

In particular, we have that $\|u(\cdot, t)\|_{L} \leqq C(1+t)^{-3 / 2}\|v(\cdot, t)\|_{L} \leqq C(1+t)^{-3 / 2}$.

PROOF. First we will prove by induction that $\left\|\left(_{(\mathrm{u})}, \mathrm{v}(\mathrm{n})\right)\right\| \leqq \tilde{\rho}$ for al1 $n=0,1,2 \ldots$ If $n=0$ this is trivial. Suppose that $\left\|{\left(u_{(n)}, v(n)\right.}_{(n)}\right\| \leqq \tilde{\rho}$. Using the definition of $u_{(n+1)}$ and $v_{(n+1)}$ we obtain

$$
\begin{aligned}
\left\|u_{(n+1)}(\cdot, t)\right\|_{H^{3}}^{2} & \left\|v_{(n+1)}(\cdot, t)\right\|_{H^{3}}^{2} \leqq 2\left(\left\|u_{o}(\cdot, t)\right\|_{H^{3}}^{2}+\left\|v_{o}(\cdot, t)\right\|_{H^{3}}^{2}\right)+ \\
& +2\left(\left\|N_{m}\left[u_{(n)}, v_{(n)}\right](\cdot, t)\right\|_{H^{3}}^{2}+\left\|N_{\sigma}\left[v_{(n)}, u_{(n)}\right](\cdot, t)\right\|_{H^{3}}^{2}\right)
\end{aligned}
$$

and

$$
\begin{aligned}
& (1+t)^{3}\left[\left\|u_{(n+1)}(\cdot, t)\right\|_{W}^{2}{ }^{1, \infty}+\left\|v_{(n+1)}(\cdot, t)\right\|_{W}^{2}, \infty\right] \leqslant \\
& \leqq 2(1+t)^{3}\left[\left\|u_{0}(\cdot, t)\right\|_{W}^{2} 1, \infty+\left\|v_{o}(\cdot, t)\right\|_{W}^{2}, \infty\right]+ \\
& +2\left[\left\|N_{m}\left(u_{(n)}, v_{(n)}\right)\right\|_{W}^{2} 3,1+\left\|N_{\sigma}\left(u(n), v_{(n)}\right)\right\|_{W}^{2} 3,1\right]
\end{aligned}
$$

From (3.5) and (3.6) we conclude that

$$
\begin{aligned}
& \left.\left\|\mid{\left(u_{(n+1)}, v_{(n+1)}\right)}\right\|^{2} \leqq 2\left\|{\left(u_{o}, v_{o}\right)}^{2}\right\|^{2}+2\left(C \tilde{\rho}^{2}\right)^{2} \|{\left(u_{(n)}, v\right.}_{(n)}\right) \|^{2} \leqq \\
& \leqq 2\left(\frac{\tilde{\rho}}{2}\right)^{2}+2\left(\frac{1}{2 \sqrt{2}}\right)^{2} \tilde{\rho}^{2}<\tilde{\rho}^{2}
\end{aligned}
$$

because our choice of $\tilde{\rho}$. This concludes the proof of our claim. For any positive integer $\mathrm{n}$ we define

$$
\left.e_{n}=\|{ }_{\left(u_{(n+1)}\right.}^{-u}(n), v(n+1)^{-v}(n)\right) \|
$$

Consequently we have $e_{n} \leq \sqrt{2} \quad \tilde{\rho}^{2} e_{n-1}$ because of lemma 3 and the above observation. By iteration it follows that $e_{n} \leqq\left(\sqrt{2} C \tilde{\rho}^{2}\right)^{n} e_{0} \leqq 2^{-n} e_{o}$. Now, let $k>n$. Using the above observation we conclude that 


$$
\left.\|\|\left(_{(k)}, v_{(k)}\right)-u_{(n)}, v_{(n)}\right) \mid \| \leqq 2^{-n+1}\left[1-2^{n-k-1}\right] e_{0} \rightarrow 0
$$

as $\mathrm{k}, \mathrm{n} \rightarrow+\infty$.

Thus, there exist a pair $(u, v)$ e X such that $\left(u_{(n)}, v(n) \rightarrow(u, v)\right.$ in $X$ as $n \rightarrow \infty$. Obviously $\|(u, v)\| \leqq \tilde{\rho}$. Thus, by lemma 3 it follows that $\left\|N_{m}[u(n), v(n)]-N_{m}(u, v]\right\|_{b} \rightarrow 0$ as $n \rightarrow \infty$ and $\left\|N_{\sigma}\left[v_{(n)}, u(n)\right]-N_{m}[v, u]\right\|_{D} \rightarrow 0$ as $n \rightarrow \infty$. Consequent $1 y(u, v)=\left(u_{0}, v_{o}\right)+\left(N_{m}[u, v], N_{\sigma}[v, u]\right)$ so that the pair $(u, v)$ is a solution of system (1.1)-(1.2).

4. LOCAL ENERGY DECAY

In this section we consider finite energy solutions of the system (1.1)-(1.2) without our previous assumptions of smallness on the initial data.

We shall concentrate our attention on the local energy $E_{\Omega}(t)$ associated with the pair $(\mathrm{u}, \mathrm{v})$ :

$$
E_{\Omega}(t)=\frac{1}{2} \int_{\Omega}\left[u_{t}^{2}+|\operatorname{grad} u|^{2}+m^{2} u^{2}+v_{t}^{2}+|\operatorname{grad} v|^{2}+\sigma^{2} v^{2}+g^{2} u^{2} v^{2}\right] d x
$$

where $\Omega$ is a bounded region of $\mathbb{R}^{3}$. In many practical situations $\Omega$ can be assumed be a bal1. We will show in this section that under suitable assumptions on the initial data of the system $(1.1)-(1.2)$ then $E_{\Omega}(t)$ approaches zero as $t \rightarrow+\infty$.

Our analysis is based on the work of C. Morawetz [4] where she studied a single nonlinear equation. First, we present an existence result: Let us consider the space $\mathrm{Y}=\mathrm{H}^{1} \oplus \mathrm{L}^{2} \oplus \mathrm{H}^{1} \oplus \mathrm{L}^{2} \quad\left(\mathbb{R}^{3}\right)$ and the matrix differential operator $\mathrm{A}$ given by

$$
A=\left(\begin{array}{cccc}
0 & I & 0 & 0 \\
\Delta-\mathrm{m}^{2} & 0 & 0 & 0 \\
0 & 0 & 0 & I \\
0 & 0 & \Delta-\sigma^{2} & 0
\end{array}\right)
$$

We can rewrite (1.1)-(1.2) with $u_{1}=u, u_{2}=u_{t}, v_{1}=v$ and $v_{2}=v_{t}$ as a system of four equations of first order in time

$$
\frac{d \Phi}{d t}=A \Phi+N(\Phi)
$$

where $\Phi=\left(u_{1}, u_{2}, v_{1}, v_{2}\right)^{\tau}, N(\Phi)=\left(0,-g^{2} u v^{2}, 0,-g^{2} v u^{2}\right)^{\tau}$ (here ()$^{\tau}$ means the transpose of $(\mathrm{)})$. Clearly $A$ is skew-adjoint with domain $D(A)=\mathrm{H}^{2} \oplus \mathrm{H}^{1} \oplus \mathrm{H}^{2} \oplus \mathrm{H}^{1}\left(\mathbb{R}^{3}\right)$.

LEMMA 5. For any $\Phi, \Psi$ e $Y$ we have

$\|\mathrm{N}(\Phi)-\mathrm{N}(\Psi)\|_{\mathrm{Y}} \leqq \mathrm{C}\left(\|\Phi\|_{Y},\|\Psi\|_{Y}\right)\|\Phi-\Psi\|_{Y}$

where $C$ is an increasing function of norms $\|\Phi\|_{Y}$ and $\|\Psi\|_{Y}$.

PROOF. Let $\Phi=\left(\mathrm{u}_{1}, \mathrm{u}_{2}, \mathrm{v}_{1}, \mathrm{v}_{2}\right)^{\tau}$ and $\Psi=\left(\tilde{\mathrm{u}}_{1}, \tilde{\mathrm{u}}_{2}, \tilde{\mathrm{v}}_{1}, \tilde{\mathrm{v}}_{2}\right)^{\tau}$ e Y. Since $\mathrm{H}^{\mathrm{j}}\left(\mathbb{R}^{3}\right)$ is an algebra then $\mathrm{N}(\Phi), \mathrm{N}(\Psi)$ e $\mathrm{Y}$. The triangle inequality implies that

$$
\begin{gathered}
\|N(\Phi)-N(\Psi)\|_{Y}^{2}=\left\|g^{2} u_{1} v_{1}^{2}-g^{2} \tilde{u}_{1} \tilde{v}_{1}^{2}\right\|_{L^{2}}^{2}+\left\|g^{2} v_{1} u_{1}^{2}-g^{2} \tilde{v}_{1} \tilde{u}_{1}^{2}\right\|_{L^{2}}^{2} \leqq 2 g^{4}\left\|\left(u_{1}-\tilde{u}_{1}\right) v^{2}\right\|_{L^{2}}^{2}+ \\
+2 g^{4}\left\|\tilde{u}_{1}\left(\tilde{v}_{1}^{2}-v_{1}^{2}\right)\right\|_{L^{2}}^{2}+2 g^{4}\left\|v_{1}\left(u_{1}^{2}-\tilde{u}_{1}^{2}\right)\right\|_{L^{2}}^{2}+2 g^{4}\left\|\left(\tilde{v}_{1}-v_{1}\right) \tilde{u}^{2}\right\|_{L^{2}}^{2}
\end{gathered}
$$


Holder's inequality followed by Sobolev's inequality give us

$\|N(\Phi)-N(\Psi)\|_{Y} \leqq C\left\|u_{1}-\tilde{u}_{1}\right\|_{H^{1}}\left[\left\|v_{1}\right\|_{H^{1}}^{2}+\left\|v_{1}\right\|_{H^{1}}\left\{\left\|u_{1}\right\|_{H^{1}}+\left\|\tilde{u}_{1}\right\|_{H^{1}}\right\}\right]+$

$$
+C\left\|v_{1}-\tilde{v}_{1}\right\|_{H^{1}}\left[\left\|\tilde{u}_{1}\right\|_{H^{1}}^{2}+\left\|\tilde{u}_{1}\right\|_{H^{1}}\left\{\left\|v_{1}\right\|_{H^{1}}+\left\|\tilde{v}_{1}\right\|_{H^{1}}\right\}\right] \leqq C\left[\|\Phi\|_{Y}+\|\Psi\|_{Y}\right]^{2}\|\Phi-\Psi\|_{Y}
$$

LEMMA 6. a) N: $D(A) \rightarrow D(A)$ and b) $\|A(N(\Phi)-N(\Psi))\|_{Y} \leqq C\left(\|\Phi\|_{Y},\|\Psi\|_{Y},\|A \Phi\|_{Y},\|A \Psi\|_{Y}\right)\|A \Phi-A \Psi\|_{Y}$ for all $\Phi, \Psi$ e $D(A)$.

PROOF. Item a) is trivial. Let $\Phi=\left(\mathrm{u}_{1}, \mathrm{u}_{2}, \mathrm{v}_{1}, \mathrm{v}_{2}\right)^{\tau}$ and $\Psi=\left(\tilde{\mathrm{u}}_{1}, \tilde{\mathrm{u}}_{2}, \tilde{\mathrm{v}}_{1}, \tilde{\mathrm{v}}_{2}\right)^{\tau}$ belonging to $D(A)$. A direct calculation gives us

$$
\begin{aligned}
&\|A(N(\Phi)-N(\Psi))\|_{Y}^{2}=g^{4}\left\|u_{1} v_{1}^{2}-\tilde{u}_{1} \tilde{v}_{1}^{2}\right\|_{H^{1}}^{2}+g^{4}\left\|v_{1} u_{1}^{2}-\tilde{v}_{1} \tilde{u}_{1}^{2}\right\|_{H^{1}}^{2} \leqq \\
& \leqq \leq g^{4}\left\|\left(u_{1}-\tilde{u}_{1}\right) v_{1}^{2}\right\|_{H^{1}}^{2}+2 g^{4}\left\|\tilde{u}_{1}\left(\tilde{v}_{1}^{2}-v_{1}^{2}\right)\right\|_{H^{1}}^{2}+2 g^{4}\left\|\left(v_{1}-\tilde{v}_{1}\right) \tilde{u}_{1}^{2}\right\|_{H^{1}}^{2}+ \\
&+2 g^{4}\left\|v_{1}\left(\tilde{u}_{1}^{2}-u_{1}^{2}\right)\right\|_{H^{1}}^{2}
\end{aligned}
$$

We use Hblder's and Sobolev's inequality to obtain

$$
\begin{aligned}
& \left\|\left(\mathrm{u}_{1}-\tilde{\mathrm{u}}_{1}\right) \mathrm{v}_{1}^{2}\right\|_{\mathrm{H}^{1}} \leqq \mathrm{C}\|\mathrm{A} \Phi-\mathrm{A} \Psi\|_{\mathrm{Y}}\|\mathrm{A} \Phi\|_{\mathrm{Y}}^{2} \\
& \left\|\tilde{u}_{1}\left(\tilde{v}_{1}^{2}-v_{1}^{2}\right)\right\|_{H^{1}} \leqq C\|A \Phi-A \Psi\|_{Y}\left[\left(\|\Phi\|_{Y}+\|\Psi\|_{Y}\right)\left(\|A \Psi\|_{Y}+\|\psi\|_{Y}\right)+\|\Psi\|_{Y}\left(\|A \Phi\|_{Y}+\|A \Psi\|_{Y}\right]\right. \\
& \left\|\left(\mathrm{v}_{1}-\tilde{\mathrm{v}}_{1}\right) \tilde{\mathrm{u}}_{1}^{2}\right\|_{\mathrm{H}^{1}} \leqq \mathrm{C}\|\mathrm{A} \Phi-\mathrm{A} \Psi\|_{\mathrm{Y}}\|\mathrm{A} \Psi\|_{\mathrm{Y}}^{2} \\
& \left\|v_{1}\left(\tilde{u}_{1}^{2}-u_{1}^{2}\right)\right\|_{H^{1}} \leqq c\|A \Phi-A \Psi\|_{Y}\left[\left(\|\Psi\|_{Y}+\|\Phi\|_{Y}\right)\left(\|A \Phi\|_{Y}+\|\Phi\|_{Y}\right)+\|\Phi\|_{Y}\left(\|A \Psi\|_{Y}+\|A \Phi\|_{Y}\right)\right]
\end{aligned}
$$

Combining the last four inequalities with (4.3) we conclude the proof of the lemma.

THEOREM 2 (Global existence). Let the initial data at time $t=0$ for the system (1.1)(1.2) belong to the subspace $\mathrm{D}(\mathrm{A})=\mathrm{H}^{2} \Theta \mathrm{H}^{1} \Theta \mathrm{H}^{2} \oplus \mathrm{H}^{1}\left(\mathbb{R}^{3}\right)$. Then, there exist a (strong) solution of (4.2) for all time $t \geqq 0$.

PROOF. According to Segal's theorem [6], lemmas 4 and 5 imply that there exists a unique local solution of (4.2) defined in a maximal interval $I=\left\{0<t<T_{\max } \leqq+\infty\right\}$ of existence. Now, let us write (1.1)-(1.2) as

$$
\begin{aligned}
& \square \mathrm{u}+\mathrm{m}^{2} \mathrm{u}=\mathrm{f} \\
& \square \mathrm{v}+\sigma^{2} \mathrm{v}=\mathrm{h}
\end{aligned}
$$

where $f=-g^{2} u v^{2}$ and $h=-g^{2} v u^{2}$. We can use the linear theory: Multiply (4.4) by $u_{t}$ and (4.5) by $v_{t}$. Next, integration in the whole space give us

$$
\begin{aligned}
& \frac{1}{2} \frac{d}{d t} \int\left[u_{t}^{2}+|\operatorname{grad} u|^{2}+m^{2} u^{2}\right] d x=\int f u_{t} d x \\
& \frac{1}{2} \frac{d}{d t} \int\left[v_{t}^{2}+|\operatorname{grad} v|^{2}+\sigma^{2} v^{2}\right] d x=\int h v_{t} d x
\end{aligned}
$$

But $\int\left(f u_{t}+h v_{t}\right) d x=-\frac{g^{2}}{2} \frac{d}{d t} \int u^{2} v^{2} d x$. Adding the identities (4.6) we conclude that

$$
E_{\infty}(t)=\frac{1}{2} \int\left[u_{t}^{2}+|\operatorname{grad} u|^{2}+m^{2} u^{2}+v_{t}^{2}+|\operatorname{grad} v|^{2}+\sigma^{2} v^{2}+g^{2} u^{2} v^{2}\right] d x=\text { Constant }
$$


in the interval I. In particular, this implies that $\|\Phi(t)\|_{Y}$ is bounded for all $t$ e I. This concludes the proof of the theorem.

REMARKS. Using essentially the same procedure as above one can prove higher regularity of the solutions provided that the initial data is more regular. If the initial data belongs to $\mathrm{H}^{\mathrm{j}+1} \oplus \mathrm{H}^{\mathrm{j}} \mathrm{HH}^{\mathrm{j}+1} \oplus \mathrm{H}^{\mathrm{j}}\left(\mathbb{R}^{3}\right)$ then the solution pair of (1.1)-(1.2) will belong to $\left[\mathrm{C}\left(\mathrm{I} ; \mathrm{H}^{\mathrm{j}+1}\left(\mathbb{R}^{3}\right)\right)\right]^{2}$.

LEMMA 7. Let $(u, v)$ be the solution of the system (1.1)-(1.2) with initial data belonging to $\left[C_{0}^{\infty}\left(\mathbb{R}^{3}\right)\right]^{4}$. Then, for any $T>0$ and $y$ e $\mathbb{R}^{3}$ we have

$$
\int_{0}^{T}\left[u^{2}(y, t)+v^{2}(y, t)\right] d t \leqq C E_{\infty}(0)
$$

where $C>0$ is independent of $T$ and $E_{\infty}(0)=E_{\mathbb{R}^{3}}(0)$ is given by (4.1) with $\Omega=\mathbb{R}^{3}$.

PROOF. Let $y \in \mathbb{R}^{3}$. For any $x \neq y$ let us denote by $r=|x-y|$ and $\frac{\partial}{\partial r}=\frac{(x-y)}{r} \operatorname{grad}$. We consider Morawetz's multiplier $M(u)=\frac{\partial u}{\partial r}+\frac{u}{r} \cdot \operatorname{Multiply}(1.1)$ by $M(u)$ and (1.2) by $M(v)$. Adding those two expressions we obtain after some calculations

$$
0=\left(\square u+m^{2} u+g^{2} u v^{2}\right) M(u)+\left(\square v+\sigma^{2} v+g^{2} v u^{2}\right) M(v)=\frac{\partial A}{\partial t}+\operatorname{div} B+D
$$

where $A=u_{t} M(u)+v_{t} M(v)$

$$
\begin{aligned}
B=\left[m^{2} u^{2}\right. & \left.+\sigma^{2} v^{2}+g^{2} u^{2} v^{2}+|g r a d u|^{2}+|\operatorname{grad} v|^{2}-u_{t}^{2}-v_{t}^{2}-\frac{u^{2}}{r^{2}}-\frac{v^{2}}{r^{2}}\right] \frac{(x-y)}{2 r}- \\
& -[M(u) \operatorname{grad} u+M(v) \operatorname{grad} v]
\end{aligned}
$$

and

$$
\mathrm{D}=\frac{1}{\mathrm{r}}\left[|\operatorname{grad} \mathrm{u}|^{2}-\mathrm{u}_{\mathrm{r}}^{2}+|\operatorname{grad} \mathrm{v}|^{2}-\mathrm{v}_{\mathrm{r}}^{2}+\mathrm{g}^{2} \mathrm{u}^{2} \mathrm{v}^{2}\right]
$$

Integration in $\mathbb{R}^{3}$ of the identity (4.8) give us

$$
\frac{d}{d t} \int A(x, t) d x-\int \operatorname{div}\left[(x-y) \frac{\left(u^{2}+v^{2}\right)}{2 r^{3}}\right] d x+\int D(x, t) d x=0
$$

Since $\mathrm{D} \geqq 0$ we obtain

$$
\frac{d}{d t} \int A(x, t) d x+2 \pi u^{2}(y, t)+2 \pi v^{2}(y, t) \leqq 0
$$

Integration from $t=0$ to $t=T>0$ gives us

$$
\int_{0}^{T}\left[u^{2}(y, t)+v^{2}(y, t)\right] d t \leqq \frac{1}{2 \pi} \int[A(x, 0)-A(x, T)] d x
$$

Let us estimate $\int A(x, t) d x$. The following simple inequalities are useful:

$$
\begin{aligned}
& \pm 2 M(u) u_{t} \leqq u_{r}^{2}+\left(\frac{u^{2}}{r}\right)_{r}+\frac{2 u^{2}}{r^{2}}+u_{t}^{2}=\operatorname{div}\left(\frac{(x-y) u^{2}}{r^{2}}\right)+u_{r}^{2}+u_{t}^{2} \\
& \pm 2 M(v) v_{t} \leqq \operatorname{div}\left(\frac{(x-y) v^{2}}{r^{2}}\right)+v_{r}^{2}+v_{t}^{2}
\end{aligned}
$$


Thus, for any $t 0$ we have

$$
\pm \int A(x, t) d x \leqq \int\left(u_{r}^{2}+u_{t}^{2}+v_{r}^{2}+v_{t}^{2}\right) d x \leqq 2 E_{\infty}(0)
$$

From (4.9) and (4.10) we obtain the conclusion of the lemma.

LEMMA 8. Let $\omega: \mathbb{R}^{3} \rightarrow \mathbb{R}$ be a $c^{1}$ function and $y \in \mathbb{R}^{3}$. Then

a) $\omega_{\tau}^{2} \leqq|\operatorname{grad} \omega(x)|^{2}-\omega_{\mathrm{r}}^{2}(\mathrm{x})$ for any $\mathrm{x}$ such that $|\mathrm{x}-\mathrm{y}|=\mathrm{r}$. Here $\omega_{\tau}(\mathrm{x})=\tau(\mathrm{x}) \cdot \operatorname{grad} \omega(\mathrm{x})$ and $\omega_{r}(x)=\frac{(x-y)}{r} \operatorname{grad} \omega(x)$ where $\tau(x)$ denotes a (unit) tangent vector at $x$.

b) $|\operatorname{grad} \omega(x)|^{2} \leqq 3 \sum^{3} \omega_{\tau_{j}}^{2}(x)$ for any $x \in \mathbb{R}^{3}$ where $\tau_{1}, \tau_{2}$ and $\tau_{3}$ are (unit) tangent vectors to the spheres $s_{j}=\left\{\xi\right.$ e $\mathbb{R}^{3}$ such that $\left|\xi_{-}-\xi_{j}\right|=\left|x-\xi_{j}\right| j=1,2,3$ respectively, for some convenient choice of $\xi_{1}, \xi_{2}$ and $\xi_{3}$.

PROOF. Given $\tau(x)$ let us choose another vector $\tau_{0}$ so that $\left\{\tau(x), \tau_{0}, \eta\right\}$ are orthonormal. Here $n$ denotes a vector in the direction of the radious $r=|x-y|$. Now, it is clear that $\omega_{\tau}^{2}+\omega_{r}^{2}(x) \leqq|\operatorname{grad} \omega(x)|^{2}$. This proves item a). Let $\xi_{j}, j=1,2,3$ and three planes $P_{j}, j=1,2,3$ so that $x$ e $P_{1} \cap P_{2} \cap P_{3}$ and their normal vectors are $x-\xi_{j}, j=1,2,3$ respectively. Let $\tau_{j}(x)$ e $P_{j}$ be (unit) tangent vectors to the spheres $s_{j}=\left\{\xi \in \mathbb{R}^{3}\right.$ such that $\left.\left|\xi_{-}-\xi_{j}\right|=\left|x-\xi_{j}\right|\right\}$ so that they are linearly independent and the angle between grad $\omega(x)$ and $\tau_{j}(x)$ is less or equal to $\pi / 2$. Then we can write $\operatorname{grad} \omega(x)$ as a linear combination of the $\tau_{j}(x)^{\prime} s, j=1,2,3$ with nonnegative coefficients. Therefore $|\operatorname{grad} \omega(x)| \leqq \sum_{j=1}^{3} \omega_{\tau_{j}}(x)$ which implies $|\operatorname{grad} \omega(x)|^{2} \leqq 3 \sum_{j=1}^{3} \omega_{\tau_{j}}^{2}(x)$.

LEMMA 9. Let $(u, v)$ be the solution of system (1.1)-(1.2) with initial data at time $t=0$ belonging to $\left[C_{0}^{\infty}\left(\mathbb{R}^{3}\right)\right]^{4}$. Let $m, \sigma \leqq 1$ and $\Omega \subseteq \mathbb{R}^{3}$ a bounded region, then for any $T>0$ we have
a) $\int_{0}^{T} \int_{\Omega}\left[|\operatorname{grad} u|^{2}+|\operatorname{grad} v|^{2}+\mathrm{g}^{2} \mathrm{u}^{2} \mathrm{v}^{2}\right] \mathrm{dxd} t \leqq \mathrm{C}(\Omega) \mathrm{E}_{\infty}(0)$
b) $\int_{0}^{T} E_{\Omega}(t) d t \leqq C(\Omega) E_{\infty}(0)$

where $\mathrm{C}(\Omega)$ is a positive constant independent of $\mathrm{T}$.

PROOF. a) We use identity (4.8). Integration in the whole space gives us, for any y e $\Omega$ :

$$
\int_{\Omega} D(x, t) d x \leqq 2 \pi\left[u^{2}(y, t)+v^{2}(y, t)\right]+\int D(x, t) d x=-\frac{d}{d t} \int A(x, t) d x
$$

Therefore, integration in time from $t=0$ to $t=T$ implies that

$$
\int_{0}^{T} \int_{\Omega} D(x, t) d x d t \leqq C E_{\infty}(0)
$$

because we have used our previous estimate $(4.10)$.

Let $d=d i a m e t e r$ of $\Omega$ and $\rho>d \geqq r=|x-y|$. Thus, from (4.11) we obtain

$$
\frac{1}{\rho} \int_{0}^{\mathrm{T}} \int_{\Omega} \mathrm{rD}(\mathrm{x}, \mathrm{t}) \mathrm{dxd} \leq \mathrm{CE}_{\infty}(0)
$$


Therefore

$$
\int_{0}^{\mathrm{T}} \int_{\Omega}\left[|\operatorname{grad} u|^{2}-\mathrm{u}_{\mathrm{r}}^{2}+|\operatorname{grad} \mathrm{v}|^{2}-\mathrm{v}_{\mathrm{r}}^{2}+\mathrm{g}^{2} \mathrm{u}^{2} \mathrm{v}^{2}\right] \mathrm{dxd} t \leqq \mathrm{C} \rho \mathrm{E}_{\infty}(0)
$$

Now, we use lemma 8 with $y=\xi_{j}, j=1,2,3$. By part a) and (4.12) we obtain

$$
\int_{0}^{\mathrm{T}} \int_{\Omega}\left\{\sum^{3}\left(\mathrm{u}_{\tau_{\mathrm{j}}^{2}}+\mathrm{v}_{\tilde{\tau}}^{2}{ }_{\mathrm{j}}\right)+\mathrm{g}^{2} \mathrm{u}^{2} \mathrm{v}^{2}\right\} \mathrm{dxdt} \leqq \mathrm{CpE}_{\infty}(0)
$$

Using part b) of lemma 8 and (4.13) we conclude the proof of part a). It remains to obtain a bound for

$$
\int_{0}^{T} \int_{\Omega}\left\{m^{2} u^{2}+u_{t}^{2}+\sigma^{2} v^{2}+v_{t}^{2}\right\} d x d t
$$

Let $\alpha>0$ and $h:[0, \infty) \rightarrow \mathbb{R}$ a $c^{\infty}$ function such that 1$\left.) h(0)=\alpha, 2\right) h \equiv 0$ for all $s \geqq \alpha$ and 3) $h^{\prime}(s)<0$ for a11 $0 \leqq s<\alpha$.

Let $y \in \mathbb{R}^{3}$ and $x \neq y$. Denote by $r=|x-y|$. First, we multiply identity (4.8) by $h(r)$ and then we integrate in space to obtain

$$
\begin{aligned}
0=2 \pi \alpha\left[u^{2}(y, t)+v^{2}(y, t)\right] & -\int h^{\prime}(r) B \cdot \frac{(x-y)}{r} d x+\frac{d}{d t} \int h(r) A(x, t) d x+ \\
& +\int h(r) D(x, t) d x
\end{aligned}
$$

The following identity can be easily verify

$$
\begin{aligned}
2 B \cdot \frac{(x-y)}{r}=g^{2} u^{2} v^{2} & +|g r a d u|^{2}+|g r a d v|^{2}-2 u_{r}^{2}-2 v_{r}^{2}+m^{2} u^{2}+\sigma^{2} v^{2}-u_{t}^{2}-\frac{u^{2}}{r^{2}}-v_{t}^{2}-\frac{v^{2}}{r^{2}}- \\
& -\frac{2 u u_{r}}{r}-\frac{2 v v_{r}}{r}
\end{aligned}
$$

Substitution of (4.15) in (4.14) and then integration in time from $t=0$ to $t=T$ implies

$$
\begin{aligned}
&-\frac{1}{2} \int_{0}^{T} h^{\prime}(r)\left[u_{t}^{2}+v_{t}^{2}+\left(\frac{1}{r^{2}}-1\right)\left(m^{2}+u^{2}+\sigma^{2} v^{2}\right)+\frac{2 u u}{r}+\frac{2 v v}{r}\right] d x d t= \\
&=2 \pi \alpha \int_{0}^{T}\left[u^{2}(y, t)+v^{2}(y, t)\right] d t-\int_{0}^{T} \int h^{\prime}(r)\left[g^{2} u^{2} v^{2}+|g r a d u|^{2}+\right. \\
&\left.+|g r a d v|^{2}-2 u_{r}^{2}-2 v_{r}^{2}\right] d x d t+\int h(r)[A(x, T)-A(x, 0)] d x+ \\
&+\int_{0}^{T} h(r) D(x, t) d x d t
\end{aligned}
$$

Now, using lemma $7,(4.10)$ and (4.11) we deduce from (4.16) the following estimate

$$
\begin{aligned}
-\int_{0}^{T} \frac{h^{\prime}(r)}{2}\left[u_{t}^{2}\right. & \left.+\left(\frac{1}{r^{2}}-1\right)\left(m^{2} u^{2}+\sigma^{2} v^{2}\right)+v_{t}^{2}+\frac{2 u u_{r}}{r}+\frac{2 v v_{r}}{r}\right] d x d t \leq C E_{\infty}(0)+ \\
& +C \operatorname{Max}_{0 \leq \xi \leq \alpha}\left|h^{\prime}(\xi)\right| \int_{0}^{T}|x-y| \leq \alpha
\end{aligned}
$$


Finally, we use (4.12) to obtain from (4.17)

$$
\begin{gathered}
-\frac{1}{2} \int_{0}^{T} \int^{\prime}(r)\left[\left(\frac{u}{r}+u_{r}\right)^{2}+\left(\frac{v}{r}+v_{r}\right)^{2}+u_{t}^{2}+v_{t}^{2}+\left(\frac{\left(m^{2}-1\right)}{r^{2}}-m^{2}\right) u^{2}+\left(\frac{\left(\sigma^{2}-1\right)}{r^{2}}-\sigma^{2}\right) v^{2}-u_{r}^{2}-\right. \\
\left.-v_{r}^{2}\right] d x d t \leqq \mathrm{CE}_{\infty}(0)+C \operatorname{Max}_{0 \leqq \xi \leqq \alpha}\left|h^{\prime}(\xi)\right| C(\Omega) E_{\infty}(0)
\end{gathered}
$$

Let us choose $\alpha=\operatorname{Min}\left\{\frac{\sqrt{\mathrm{m}^{2}-1}}{\sqrt{2} \mathrm{~m}}, \frac{\sqrt{\sigma^{2}-1}}{\sqrt{2} \sigma}\right\}=\alpha_{0}$. Thus, if $r=|x-y| \leqq \alpha$ it follows that

$$
-\int_{0}^{T} \int_{|x-y| \leqq \alpha} h^{\prime}(r)\left[u_{t}^{2}+v_{t}^{2}+m^{2} u^{2}+\sigma^{2} v^{2}\right] d x d t \leqq C(\Omega) E_{\infty}(0)
$$

In particular

$$
-\int_{0}^{T} \int_{|x-y| \leqq \alpha / 2} \frac{h^{\prime}(r)}{2}\left[u_{t}^{2}+v_{t}^{2}+m^{2} u^{2}+\sigma^{2} v^{2}\right] d x d t \leqq C(\Omega) E_{\infty}(0)
$$

or

$$
B \int_{0}^{T} \int_{|x-y| \leqq \alpha / 2}\left[u_{t}^{2}+v_{t}^{2}+m^{2} u^{2}+\sigma^{2} v^{2}\right] d x d t \leqq C(\Omega) E_{\infty}(0)
$$

where $\beta=\operatorname{Inf}_{r \leq \alpha / 2}\left(-h^{\prime}(r)\right)>0$

Combining part a) with (4.19) we obtain that

$$
\int_{0}^{T} E_{\alpha}(t) d t \leqq C(\Omega) E_{\infty}(0)
$$

where $\Omega_{\alpha}=\{x,|x-y| \leqq \alpha / 2\}$. Since $\Omega$ is a bounded region we can cover it by a finite number of such balls. This implies part b).

THOEREM 3 (Decay of local energy). Let $(u, v)$ be the solution of system (1.1)-(1.2) with initial data belonging to $\left[C_{0}^{\infty}\left(\mathbb{R}^{3}\right)\right]^{4}$ and $m, \sigma \geqq 1$. Let $\Omega \subseteq \mathbb{R}^{3}$ be a bounded region, then

a) $\operatorname{Lim}_{t \rightarrow+\infty} \int_{\Omega} u^{2}(x, t) d x=\operatorname{Lim}_{t \rightarrow+\infty} \int_{\Omega} v^{2}(x, t) d x=0$ and $\left.b\right) \underset{t \rightarrow+\infty}{\operatorname{Lim}} E_{\Omega}(t)=0$

PROOF. Let $\mathrm{T}>0$. We know by lemma 7 that

$$
\int_{0}^{T} \int_{\Omega}\left(u^{2}+v^{2}\right) d x d t \leqq C(\Omega) E_{\infty}(0)
$$

Letting $\mathrm{T} \rightarrow+\infty$ we obtain

$$
\int_{0}^{\infty} \int_{\Omega}\left(u^{2}+v^{2}\right) d x d t<+\infty
$$

Let $G(t)=\int_{\Omega}\left(u^{2}+v^{2}\right) d x$. We also have 


$$
\left|\frac{d}{d t} G(t)\right|=\left|2 \int_{\Omega}\left(u u_{t}+v v_{t}\right) d x\right| \leqq \int_{\Omega}\left(u^{2}+u_{t}^{2}+v^{2}+v_{t}^{2}\right) d x \leqq C E_{\infty}(0)
$$

From (4.20) and (4.21) it follows that $\operatorname{Lim}_{t \rightarrow+\infty} G(t)=0$. Consequent $1 y, \operatorname{Lim}_{t \rightarrow \infty} u_{\Omega} \mathrm{u}^{2} \mathrm{dx}=\operatorname{Lim} \int_{\mathrm{t} \rightarrow \infty} \mathrm{v}^{2} \mathrm{dx}=0$. Let us look back to the inequality (4.19) and let us fix $\alpha_{1}, \alpha_{2}$ so that $0<\alpha_{1}<\alpha_{2}<\alpha_{0}$. We define $F(t)$ as

$$
F(t)=\int_{\alpha_{1}}^{\alpha_{2}} E_{\alpha}(t) d \alpha
$$

where $\Omega_{\alpha}=\left\{x \in \mathbb{R}^{3} /|x-y| \leqq \alpha / 2\right\}$. Integration in time of (4.22) implies

$$
\int_{0}^{\mathrm{T}} F(t) \mathrm{dt}=\int_{\alpha_{1}}^{\alpha_{2}} \mathrm{~d} \alpha \int_{0}^{\mathrm{T}} \mathrm{E}_{\Omega_{\alpha}}(t) \mathrm{d} t \leqq \mathrm{CE}_{\infty}(0)\left(\alpha_{2}-\alpha_{1}\right)
$$

Therefore $\int_{0}^{\infty} F(t) d t<+\infty$. A simple calculation shows that

$$
\left|\frac{d}{d t} F(t)\right| \leqq\left.\frac{1}{2}\right|_{\alpha_{1} \leqq}|x-y| \leqq \alpha_{2}\left(u_{r}^{2}+u_{t}^{2}+v_{r}^{2}+v_{t}^{2}\right) d x \leqq E_{\infty}(0)
$$

which together with the above observations implies that $\operatorname{Lim}_{t \rightarrow+\infty} E_{\Omega}(t)=0$.

\section{REFERENCES}

1. SEGAL, I. Nonlinear Partial Differential Equations in Quantum Field Theory, Proc. Symp. Appl. Math. AMS, 17, (1965), 210.226.

2. JURGENS, K. Nonlinear Wave Equations, Lecture Notes, University of Colorado, March 1970.

3. MAKHANKOV, V.G. Dynamics of Classical Solutions in Integrable Systems, Physics Reports (Section C of Physics Letters) 35 (1), (1978), 1-128.

4. MORAWETZ, C. Time Decay for the Nonlinear Klein-Gordon Equation, Proc. Royal Soc. London A306, (1968), 291-296.

5. STRAUSS, W.A. Decay of Solutions of Hyperbolic Equations with Localized Nonlinear Terms, Ist. Naz. di Alta Mat., Symposia Mat., vol. VII, (1971), 339-355.

6. REED, M. Abstract Non-Linear Wave Equations, Lecture Notes in Math., 507, SpringerVerlag, (1976).

7. STRAUSS, W.A. Decay and Asymptotics for $\square \mathrm{u}=\mathrm{F}(\mathrm{u})$, J. Funct. Anal., 2, (1968), 409457. 


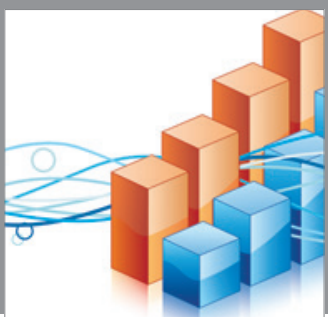

Advances in

Operations Research

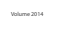

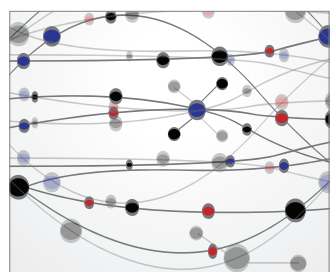

\section{The Scientific} World Journal
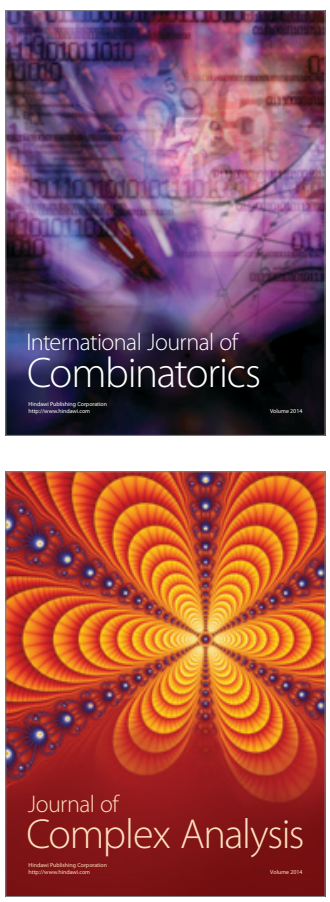

International Journal of

Mathematics and

Mathematical

Sciences
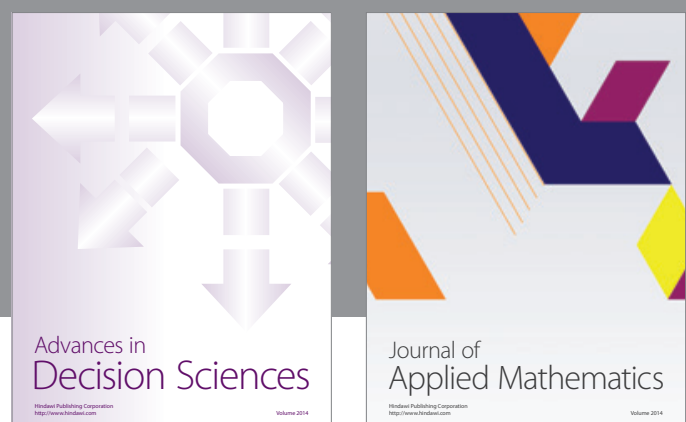

Journal of

Applied Mathematics
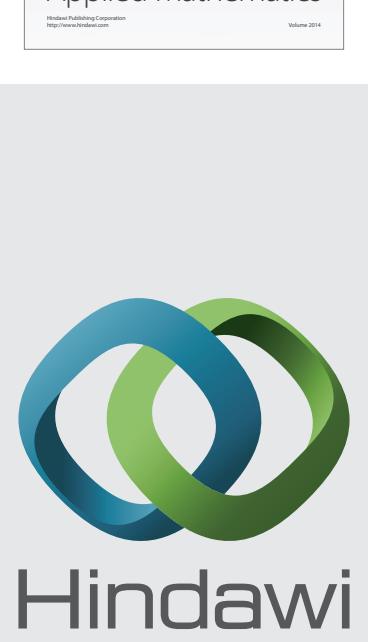

Submit your manuscripts at http://www.hindawi.com
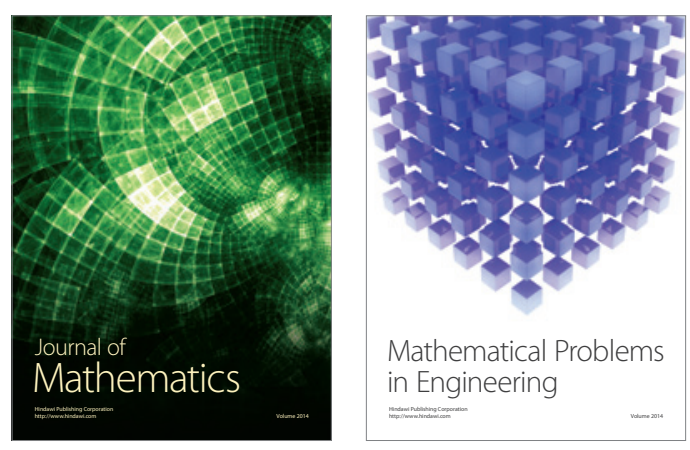

Mathematical Problems in Engineering
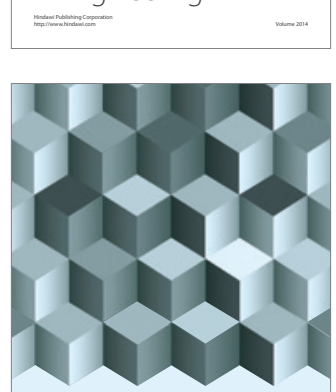

Journal of

Function Spaces
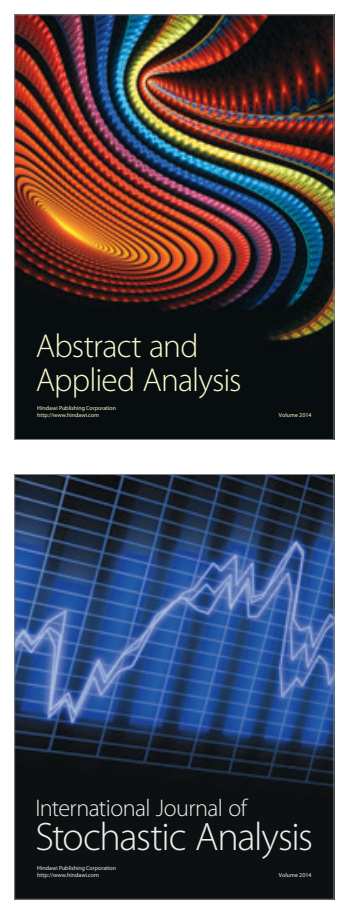

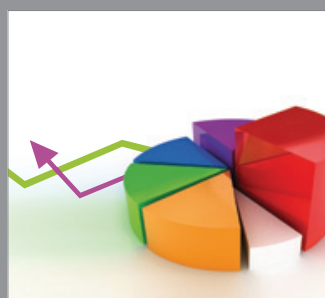

ournal of

Probability and Statistics

Promensencen
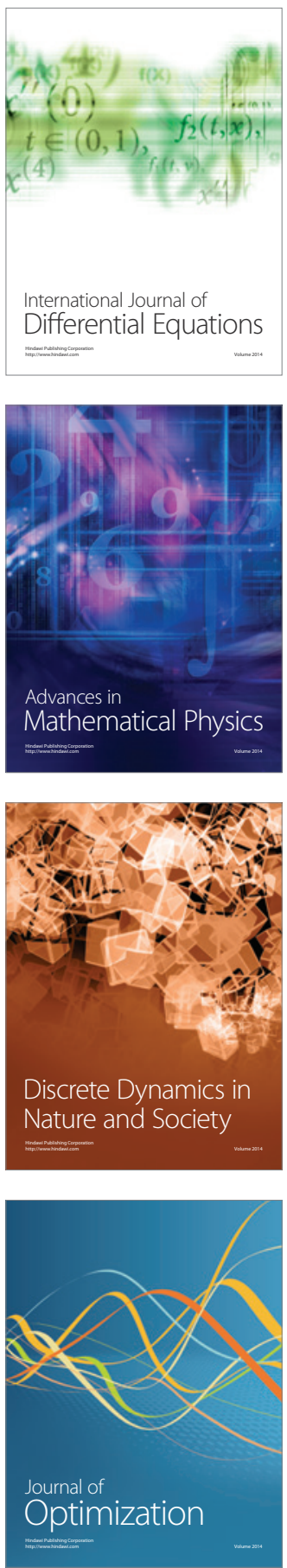